Review

\title{
An Up-Date of the Muscle Strengthening Exercise Effectiveness in Postmenopausal Women with Osteoporosis: A Qualitative Systematic Review
}

\author{
Jose Luis Alonso Pérez ${ }^{1,2,3, *}$, Sebastián Martín Pérez ${ }^{1,3}{ }^{(0)}$, Andrea Battaglino ${ }^{4}$, Jorge H. Villafañe ${ }^{4, *}$, \\ Alexandra Alonso-Sal ${ }^{1,2,3}$ and Eleuterio A. Sánchez Romero ${ }^{1,2, *(D)}$ \\ 1 Musculoskeletal Pain and Motor Control Research Group, Faculty of Health Sciences, Universidad Europea \\ de Madrid, 28670 Madrid, Spain; sebastian.martin@universidadeuropea.es (S.M.P.); \\ alexandraalonsosal@hotmail.com (A.A.-S.) \\ 2 Department of Physiotherapy, Faculty of Biomedical and Health Sciences, Universidad Europea de Madrid, \\ Tajo, s/n, Urbanización El Bosque, Villaviciosa de Odón, 28670 Madrid, Spain \\ 3 Musculoskeletal Pain and Motor Control Research Group, Faculty of Health Sciences, Universidad Europea \\ de Canarias, C/Inocencio García 1, Tenerife, Canary Islands, 38300 La Orotava, Spain \\ 4 IRCCS Fondazione Don Carlo Gnocchi, Piazzale Morandi 6, 20148 Milan, Italy; \\ andrea.battaglino@edu.unito.it \\ * Correspondence: joseluis.alonso@universidadeuropea.es (J.L.A.P.); mail@villafane.it (J.H.V.); \\ eleuterio.sanchez@universidadeuropea.es (E.A.S.R.)
}

Citation: Alonso Pérez, J.L.; Martín Pérez, S.; Battaglino, A.; Villafañe, J.H.; Alonso-Sal, A.; Sánchez Romero, E.A. An Up-Date of the Muscle Strengthening Exercise Effectiveness in Postmenopausal Women with Osteoporosis: A Qualitative Systematic Review. J. Clin. Med. 2021, 10, 2229. https://doi.org/10.3390/ jcm10112229

Academic Editors: Martina Rauner, Elizabeth Winter and Andrea Burden

Received: 27 March 2021

Accepted: 19 May 2021

Published: 21 May 2021

Publisher's Note: MDPI stays neutral with regard to jurisdictional claims in published maps and institutional affiliations.

Copyright: (c) 2021 by the authors. Licensee MDPI, Basel, Switzerland. This article is an open access article distributed under the terms and conditions of the Creative Commons Attribution (CC BY) license (https:// creativecommons.org/licenses/by/ $4.0 /)$.

\begin{abstract}
Background: Osteoporosis (OP) is a systemic disease that is characterized by decreased bone density and quality. Purpose: The purpose of this systematic review was to determine the effects of muscle strengthening exercise in postmenopausal women with OP. Methods: A literature search was conducted systematically in MEDLINE, CINAHL, EMBASE databases for human studies up to 31 March 2021. Two researchers screened the articles against predefined inclusion criteria; a third resolved discrepancies. Articles were included if they assessed the effects of muscle strengthening exercise in postmenopausal women with OP. The protocol for this systematic review was registered on PROSPERO (CRD42021207917) and a qualitative systematic review was carried out following the PRISMA statement. Methodological quality was evaluated through the scientific validity scales PEDro. Finally, RTCs and NRCTs risk of bias was assessed with the Cochrane risk of bias tool (Risk of Bias-ROB 2.0) and ROBINS-1, respectively. Results: A total of 16 studies (1028 subjects) that met the different eligibility criteria previously established were selected. There is evidence of good methodological quality and a low to moderate risk of bias that supports that muscle strengthening exercise alone or in combination with other therapeutic modalities improves BMD $(9, n=401)$ in proximal femur and lumbar vertebra body, muscle strength $(10, n=558)$, balance $(4, n=159)$, functionality $(7, n=617)$, and quality of life $(5, n=291)$. Conclusions: Exercise programs focused on muscle strengthening have benefits for all variables studied in postmenopausal women with OP.
\end{abstract}

Keywords: exercise; women; osteoporosis

\section{Introduction}

Osteoporosis (OP) represents a pathology of important health implications, which identifies its clinical significance in the fracture that occur as a consequence of increased bone fragility [1]. In 2010, it was estimated that 22 million women aged over 50 years old in the EU had osteoporosis using the diagnostic criterion of the WHO [1]. The high societal and personal cost of osteoporosis pose challenges to publich health and physician, particularly since most patients with osteoporosis remain untreated [2].

The standard treatment is fundamentally pharmacological and aims to reduce the incidence of fractures through the interruption of the resolution mechanism [3]. It appears 
that although the benefits outweigh the risks, the association between the combination of sequential antiresorptive/anabolic pharmacotherapeutic cycles and the reduction of fracture risk using aminobisphosphonates, selective estrogen receptor modulators, denosumab and teriparatide has not been demonstrated. However, when combined with exercise this would help maintain bone architecture thanks to increased bone mass and muscle strength $[4,5]$. Muscle strengthening exercise would be effective because it is believed to increase muscle mass and decrease fat mass, especially in osteoporotic menopausal women in whom, by stimulating osteogenesis, it would reduce falls and fractures [6,7].

In contrast, Ashe et al. [8], Sanudo et al. [9] and Asikainen et al. [10] have respectively highlighted the lack of efficacy of resistance exercise in elderly women, pointing out a nonsignificant positive effect on body loss in resistance protocols alone and showing how even the combination of aerobic and resistance training does not lead to improvements in terms of BMD, muscle strength, flexibility and coordination, even in programs lasting more than one year. In addition, Benedetti et al. [4] has reported the low efficacy of long-term muscle strengthening exercise in elderly women in terms of cortical volumetric bone mineral density when performed with own body weight or resistance. Perhaps this is due to the diversity of the exercise used in the research detailed in the existing literature, and to the different nomenclatures used: physical exercise, exercise, resistance training, strength training, weight-bearing exercise training, resistance training programs, land exercise program, aquatic exercise program, physical activity program, circuit training, high-intensity resistance and impact training, adapted physical activity exercise protocol, progressive load training, strengthening exercises and multicomponent training.

Due to the diversity of studies found in the literature that clearly indicate the effects of muscle strengthening exercise on bone architecture, but also the existence of other studies that deny a positive effect, the purpose of this systematic review was to present an update of studies assessing the effects of muscle strengthening exercise in postmenopausal women with OP.

\section{Materials and Methods}

\subsection{Data Source and Search Strategy}

This is a systematic literature review of studies investigating or updating the effects of muscle strengthening exercise in postmenopausal women with OP Preferred Reporting Items for Systematic Review and Meta-Analyses (PRISMA) guidelines were followed during the design, search and reporting stages of this systematic review. The protocol for this systematic review was registered on PROSPERO (CRD42021207917).

\subsection{Search Strategy}

Independent researchers (J.L.A.P., S.M.P. and A.B.) conducted a qualitative systematic review following the PRISMA statement by introducing the keywords "Osteoporosis, Postmenopausal", "Resistance Training", "Exercise", and "Isometric Contraction" as well as the free terms "Strength Training", "Musculoskeletal", "Exercise" and "Strength" combined with the Booleans "AND" and "OR" in metasearch engines Cochrane Library Plus and TripDataBase and electronic databases Pubmed (MEDLINE) and Physiotherapy Database (PEDro), Table 1, search equations.

\subsection{Eligibility Criteria}

Eligibility criteria were: (1) Randomized and non-randomized clinical trials, (2) published full text, (3) without restriction in language (4) between 1 January 2005 and 31 March 2021, (5) in which participate postmenopausal women with OP over 40 years (6) in an exercise program based on muscle strengthening exercise combined or not with other modalities.

\subsection{Data Extraction}

All relevant articles from the aforementioned datasets were identified by two reviewers who conducted the data extraction independently (J.L.A.P. and S.M.P.). A third author (A.B.) 
resolved discrepancies. Reviewers were not masked to any pieces of information regarding the authors, the journal or the outcomes for each article reviewed. A standardized form was used to extract data concerning study design, number and mean age of participants, year and country of publication, setting, exercise program involved, follow-up timing, clinical outcome measures and reported findings. The form was developed according to the directions of the Cochrane Handbook for Systematic Reviews of InterventionsVersion 5.1.0. This form was pilot-tested for reliability using a representative sample of the studies to be reviewed.

\subsection{Outcome Measure}

The primary outcomes was the change of BMD at lumbar spine and femoral bone regions, muscle strength, balance, functionality and quality of life between baseline and follow-up.

\subsection{Quality Assessment}

All the articles that met the eligibility criteria were independently assessed by two independent authors (J.L.A.P. and S.M.P.) for methodological quality with the Physiotherapy Evidence Database (PEDro) Scale and for risk of bias using Cochrane Collaboration's Risk of Bias (ROB.2.0) for RCTs and Risk of Bias in Non-randomized Studies of Interventions (ROBINS-1) for NRCTs. Disagreements were solved by discussion including a third author (A.B.) until a consensus was reached. We classified the methodological quality as follows: $\geq 7$ = high, 5-6 = moderate, and $<5=$ low and RCTS risk of bias as "high" risk of bias, "unclear" or "low" and NRCTs as "low", "moderate", "serious" and "critical".

\section{Results}

\subsection{Study Selection}

Via the databases search, 172 articles have been identified. Of these, after first screening based on title, abstracts and duplicates, 46 articles have been submitted to a second screening. After the full text reading, according to out exclusion criteria, 16 studies were eligibility for this review, with a total amount of 1028 patient (Figure 1).

\subsection{Study Characteristics and Quality Assessment}

The review included 14 RCCTs and 2 NRCCTs, whose characteristics are collected in Table 1. The methodological quality evaluation, based on PEDro Scale, reports an average score of 6/10 (PEDro Table). The risk of bias analysis, using RoB 2.0 for RCTs and ROBINS-1 for NRCTs, showed a low to moderate risk and a critical risk respectively. 

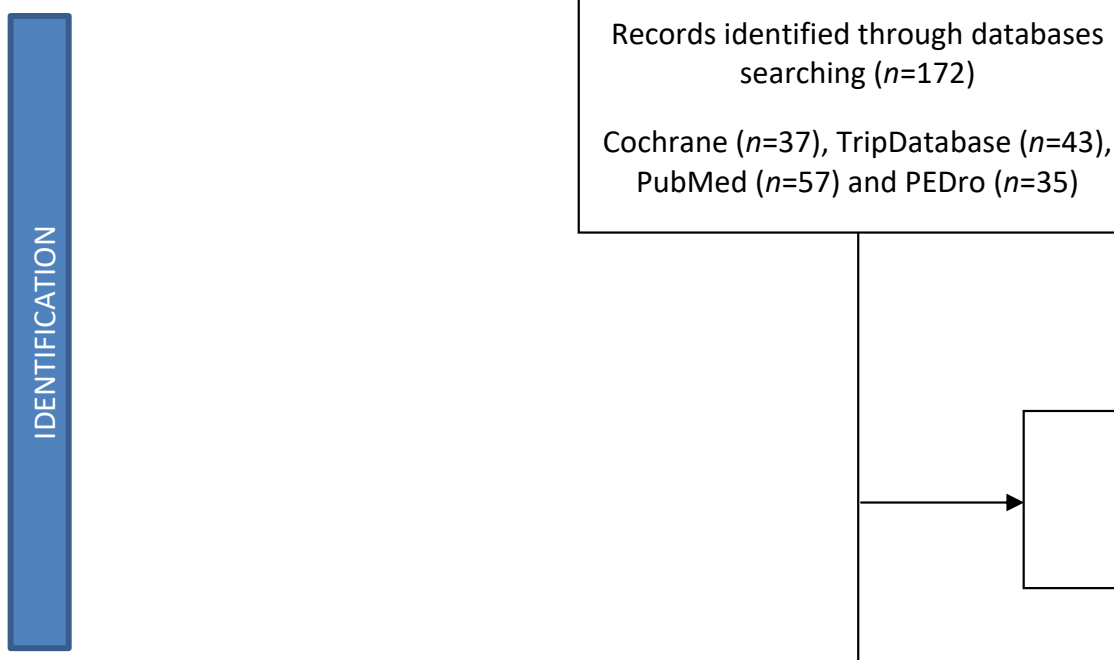

Records screened by title

$(n=172)$

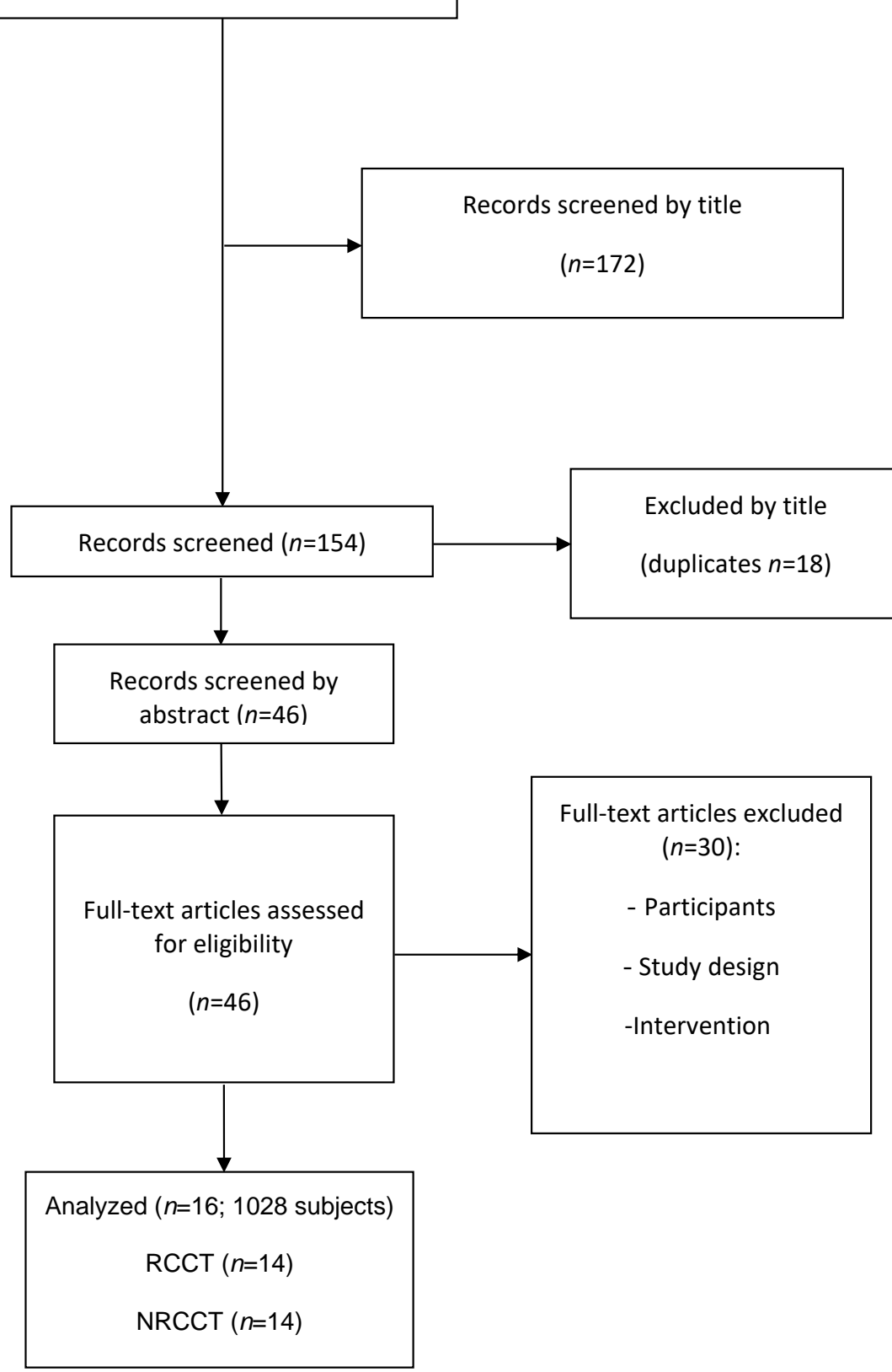

Figure 1. PRISMA Flow diagram.

\section{Summary of Results}

\subsection{Association between Exercise Therapy and Bone Quality}

Nine of the 16 studies included in this review investigated the effects of exercise on bone quality. In the tested districts (femoral neck, lumbar spine, tibia) were found a statistically significant correlation between Bone Mineral Density and BMC with a 10 to 52 week exercise protocol (Table 1) [11-19]. However, Brentano and Ashe report absence of modifications and changes not statistically significant in this outcome measure $[8,20]$. 
Table 1. Characteristics of included studies.

\begin{tabular}{|c|c|c|c|c|c|c|}
\hline Author, Year & Participants & Outcome Measures & Intervention & Results & Conclusions & PEDro Score \\
\hline $\begin{array}{l}\text { Marini et. Al (2019) } \\
\text { [21] }\end{array}$ & $\begin{array}{l}n=44 \text { women }[22 \text { experimental } \\
\text { group (APA), } 18 \text { control group } \\
\text { (CG)]. } \\
\text { Inclusion criteria: } \\
\text { 1. Post-menopausal } \\
\text { 2. } \quad \text { Agemen from } 60 \text { to } 75 \\
\text { 3. OP verified by dual } \\
\text { energy X-ray } \\
\text { absorptiometry } \\
\text { W. With or without } \\
\begin{array}{l}\text { pharmacological therapy } \\
\text { for OP }\end{array} \\
\text { 5. One or more vertebral } \\
\begin{array}{l}\text { fractures verified by } \\
\text { radiography }\end{array}\end{array}$ & 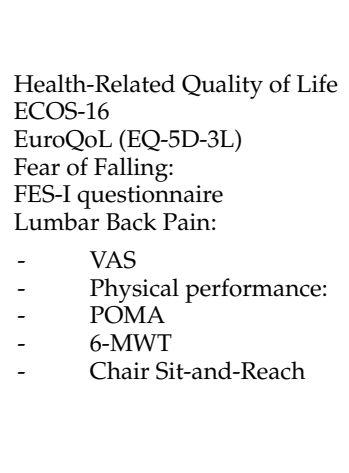 & $\begin{array}{l}\text { Protocol duration: } 6 \text { months } \\
\text { Frequency: } 2 \text { times per week } \\
\text { Session: } 1 \mathrm{~h} \\
\text { IG: Supervised } \\
\text {-Warm-up: } 15 \text { min; multi-articular exercises, } \\
\text { focus on joint mobilization, balance and } \\
\text { postural control during walking. } \\
\text {-Workout: } 35 \text { min; resistance bodyweight } \\
\text { exercises (isometric and dynamic). } \\
\text {-Cool down: } 10 \text { min; Stretching, Exercise in an } \\
\text { upright and supine static position, holding a } \\
\text { stretch position for up to } 30 \mathrm{~s} . \\
\text { Drug exposure (\% allocated subject): IG } 100 \% \\
\text { (Bisphosphonates) }\end{array}$ & $\begin{array}{l}\text { Adherence: } 75.8 \%(56.4-97.8 \%) \\
\text { ECOS-16: APA group }-0.5 \pm 0.5, p=0.001 * \\
\text { CG }+0.0 \pm 0.3, \mathrm{~ns} \\
\text { EuroQoL VAS: APA }+6.0 \pm 16.6, \mathrm{~ns} \text {; CG }+1.9 \\
\pm 12.1, \mathrm{~ns} \\
\text { FES-I: APA }-4.7 \pm 7.4, p=0.006 * ; \mathrm{CG}+0.9 \\
\pm 2.5, \mathrm{~ns} \\
\text { Lumbar back pain VAS: APA }-1.2 \pm 2.6 \\
p=0.029 * \text { CG }+0.3 \pm 3.3, \mathrm{~ns} \\
\text { Tinetti scale: APA }+2.8 \pm 5.2, p=0.003 * \text {; } \\
\text { CG }-0.7 \pm 2.4 . \mathrm{ns} \\
\text { 6MWT: APA }+52.2 \pm 42.1, p<0.001 * ; \\
\text { CG }-8.5 \pm 45.2, \mathrm{~ns} \\
\text { Chair Sit-and-Reach right } / \text { left: APA }+6.5 \text { and } \\
+7.3, p=0.002 * / 0.001 * ; \mathrm{CG}-0.6 \text { and }-0.2, \mathrm{~ns} \\
{ }^{*} \text { whitin group difference } p<0.05\end{array}$ & $\begin{array}{l}\text { The feasibility, the safety and } \\
\text { the positive effect of the } \\
\text { proposed exercise protocol on } \\
\text { quality of life, fear of falling, } \\
\text { balance and functional exercise } \\
\text { capacity show that APA } \\
\text { programs should be extended } \\
\text { also to patients whit OP and a } \\
\text { history of vertebral fracture. }\end{array}$ & $6 / 10$ \\
\hline $\begin{array}{l}\text { Koevska et al. (2019) } \\
\text { [22] }\end{array}$ & $\begin{array}{l}n=92 \text { women } \\
\text { Inclusion criteria: } \\
-\quad \text { Diagnosed OP } \\
-\quad \text { Total t-score }-1,5 \mathrm{SD} \text { to } \\
-2.5 \mathrm{SD} \text {, determined } \\
\text { with X-ray DXA } \\
\text { densitometry. }\end{array}$ & $\begin{array}{l}\text { Quality of life } \\
\text { QUALEFFO-41 }\end{array}$ & $\begin{array}{l}\text { Protocol duration: } 12 \text { months } \\
\text { Frequency: } 3 \text { times per week } \\
\text { IG: Exercise and physical modalities } \\
\text { (interferent currents and magnetic therapy for } \\
3 \text { week, each day with a weekend break). } \\
\text { CG 1: Exercise } \\
\text { CG 2: No exercise } \\
\text { Exercise: } 5 \text { to } 8 \text { times } \\
\text {-Respiratory } \\
\text {-Strengthening of the paraverterbral muscles, } \\
\text { upper and lower extremities muscles, } \\
\text { abdominal muscle } \\
\text {-Active exercise for maintaining the range of } \\
\text { motion of the joints of upper and lower } \\
\text { extremities and spine } \\
\text {-Balance } \\
\text { Drug exposure (\% allocated subject): IG } 100 \% \text {; } \\
\text { CG 100\% (Bisphosphonates, calcium and } \\
\text { vitamin D) }\end{array}$ & $\begin{array}{l}\text { Pain: III vs. I, } 59.3 \pm 21.3 \text { vs. } 40.87 \pm 20.6 \\
(p=0.004 *) \\
\text { III vs. II, } 59.3 \pm 21.3 \text { vs. } 31.0 \pm 23.2 \\
\left(p<0.0001^{*}\right) \\
\text { Physical function: III vs. I, } 41.8 \pm 19.3 \text { vs. } \\
19.95 \pm 13.3\left(p<0.0001^{*}\right) \\
\text { III vs. II, } 41.8 \pm 19.3 \text { vs. } 19.99 \pm 15.4 \\
(p<0.0001 *) \\
\text { Social Life: III vs. I, } 67.06 \pm 27.9 \text { vs. } 34.8 \pm \\
19.9(p<0.0001 *) \\
\text { III vs. II, } 67.06 \pm 27.9 \text { vs. } 27.65 \pm 21.64 \\
\left(p<0.0001^{*}\right) \\
\text { Health perception: III vs. I, } 78.2 \pm 21.2 \text { vs. } \\
45.88 \pm 22.1(p<0.0001 *) \\
\text { III vs. III } 78.2 \pm 21.2 \text { vs. } 41.5 \pm 21.9 \\
\left(p<0.000^{*}\right) \\
*^{*} \text { whitin group difference } p<0.05\end{array}$ & $\begin{array}{l}\text { The exercise program for OP } \\
\text { has significantly improved the } \\
\text { quality of life in patients after } \\
\text { one year of practicing in all } \\
\text { four domains: pain, physical } \\
\text { activities and mobility, social } \\
\text { activities and perception about } \\
\text { general health condition. }\end{array}$ & $8 / 10$ \\
\hline
\end{tabular}


Table 1. Cont.

\begin{tabular}{|c|c|c|c|c|c|c|}
\hline Author, Year & Participants & Outcome Measures & Intervention & Results & Conclusions & PEDro Score \\
\hline $\begin{array}{l}\text { Çergel et al. (2019) } \\
\text { [23] }\end{array}$ & 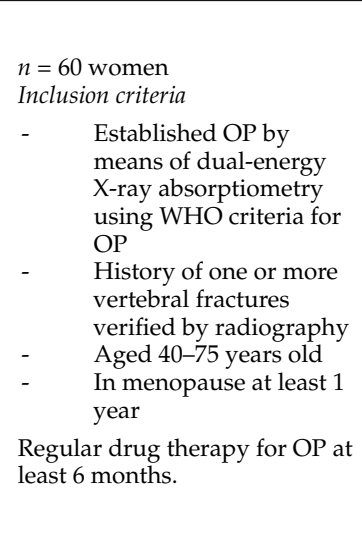 & $\begin{array}{l}\text { Spinal pain } \\
\text {-VAS } \\
\text { Back extensor muscle Strength } \\
\text { - Hand dynamometer } \\
\text { Trunk muscle endurance } \\
\text { - Timed Loaded Standing test } \\
\text { Thoracic kyphosis } \\
\text { - Digital inclinometer } \\
\text { Functional mobilty } \\
\text { - Time Up and Go test (TUG) } \\
\text { Health-related quality of life } \\
\text { - QUALEFFO-41 }\end{array}$ & $\begin{array}{l}\text { Protocol duration: } 6 \text { weeks } \\
\text { Frequency: } 3 \text { times per week } \\
\text { IG: Supervised exercise group (SE) with full } \\
\text { supervision of physiatrist. } \\
\text { CG1: Home-based exercise group (HE) with } \\
\text { instructional booklet and asked to apply the } \\
\text { program at home. } \\
\text { CG2: Daily life activities } \\
\text {-Workkout: Back extensor Strengthening } \\
\text { exercises } \\
\text { In prone position: trunk extension, alternate } \\
\text { arm raises, opposing arm and legs } \\
\text { On the hands and knees position: opposing } \\
\text { arm and leg raises. } \\
\text { I-II weeks: } 3 \text { set of } 8 \text { rep } \\
\text { III-IV weeks: } 3 \text { set of } 10 \text { rep } \\
\text { V-VI weeks: } 3 \text { set of } 12 \text { rep } \\
\text { Drug exposure (\% allocated subject): IG } 100 \% \text {; } \\
\text { CG 100\% (Bisphosphonates) at least } 6 \text { months }\end{array}$ & 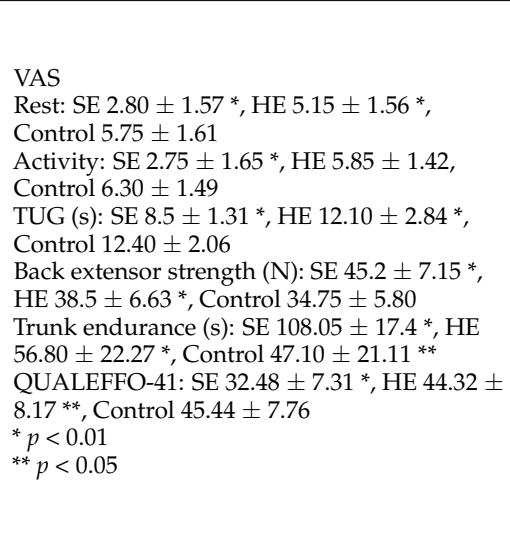 & $\begin{array}{l}\text { This study demonstrated that } \\
\text { short-term supervised back } \\
\text { extensor strengthening training } \\
\text { is superior to home-based } \\
\text { program in terms of spinal } \\
\text { pain, back extensor muscle } \\
\text { stgrength, trunk endurance, } \\
\text { functional mobility, and QoL } \\
\text { for postmenopausal } \\
\text { osteoporotic women with } \\
\text { vertebral fractures. }\end{array}$ & $6 / 10$ \\
\hline $\begin{array}{l}\text { Watson et al. (2019) } \\
\text { [19] }\end{array}$ & $\begin{array}{l}n=51 \text { women } \\
\text { Inclusion criteria } \\
\text { - Women older than } 58 \text { years } \\
\text { - Low bone mass (T-score }< \\
-1.0 \text { at the hip and/or spine). }\end{array}$ & $\begin{array}{l}\text { Magnitude of kyphosis: } \\
-\quad \text { DXA } \\
-\quad \text { Inclinometer and } \\
\quad \text { flexicurve } \\
\text { Lateral vertebral assessment } \\
\text { (LVA) and Vertebral body } \\
\text { morphology: } \\
\text { - DXA and Cobb angle }\end{array}$ & $\begin{array}{l}\text { Protocol duration: } 8 \text { months } \\
\text { Frequency: } 2 / \text { week } \\
\text { Session: } 30 \text { min } \\
\text { Intervention group (HiRIT): Supervised } \\
\text { Warm-up: } 2 \text { sets of deadlift at } 50 \% \text { to } 7 \% \text { 1RM } \\
\text { First month: } \\
\text { Body weight and low-load exercise variants, } \\
\text { with focus on progressively learning the } \\
\text { movement patterns. } \\
4 \text { fundamental exercise within } 2 \text { months } \\
\text { Remainder intervention period: } \\
\text { Resistance exercise (deadlift, overhead press, } \\
\text { back squat) } \\
\text { Training: } 5 \text { sets of } 5 \text { repetition } \\
\text { Intensity: > } 80 \% \text { to } 85 \% 1 \mathrm{RM} \\
\text { Drug exposure (\% allocated subject): IG } 100 \% \text {; } \\
\text { CG 100\% (Bisphosphonates, calcium and } \\
\text { vitamin D) }\end{array}$ & $\begin{array}{l}\text { Height }(\mathrm{cm}): \text { CON }-0.1 \pm 0.6, \text { HiRIT }+0.2 \pm \\
0.6, p=0.140 \\
\text { Inclinometer }\left(^{\circ}\right) \\
\quad \quad \text { Relaxed standing: CON }-4.2 \pm 6.7^{*}, \\
\quad \text { HiRIT }-4.7 \pm 6.3^{*}, p=0.779 \\
\quad \text { Standing tal: } \mathrm{CON}-2.0 \pm 8.1, \text { HiRIT } \\
\quad-5.3 \pm 7.1^{*}, p=0.167 \\
\text { Flexicurve kyphosis index }\left(^{\circ}\right): \text { CON }-1.9 \pm \\
2.4^{*}, \text { HiRIT }-2.1^{*} \pm 2.2^{*}, p=0.819 \\
\text { Cobb endplate angle }\left({ }^{\circ}\right): \text { CON }-0.6 \pm 4.3, \\
\text { HiRIT }+0.4 \pm 4.4, p=0.631 \\
\text { Cobb body angle }\left({ }^{\circ}\right): \text { CON }+0.5 \pm 4.5, \text { HiRIT } \\
-1.0 \pm 4.5, p=0.276 \\
{ }^{*} \text { whitin group difference } p<0.05\end{array}$ & $\begin{array}{l}\text { Observations from the } \\
\text { LIFTMOR trial indicate that } \\
\text { brief, twice-weekly, supervides } \\
\text { HiRIT exercise for } 8 \text { months did } \\
\text { not cause fragility fractures and } \\
\text { improved thoracic kyphosis in } \\
\text { postmenopausal women with } \\
\text { low to very low bone mass. }\end{array}$ & $7 / 10$ \\
\hline
\end{tabular}


Table 1. Cont.

\begin{tabular}{|c|c|c|c|c|c|c|}
\hline Author, Year & Participants & Outcome Measures & Intervention & Results & Conclusions & PEDro Score \\
\hline $\begin{array}{l}\text { Watson et al. (2018) } \\
\text { [24] }\end{array}$ & $\begin{array}{l}n=101 \text { women } \\
\text { Mean age: } 65 \pm 5 \\
\text { Inclusion criteria } \\
\text { - Women older than } 58 \text { years } \\
\text { - Low bone mass (T-score }< \\
-1.0 \text { at the hip and/or spine). }\end{array}$ & $\begin{array}{l}\text { Bone measures } \\
\text {-Femoral neck BMD } \\
\text {-Lumbar spine BMD } \\
\text {-QUS } \\
\text {-Calcaneal BUA } \\
\text {-SOS } \\
\text {-SI } \\
\text { Physical performance } \\
\text {-LES } \\
\text {-BES } \\
\text {-TUG } \\
\text {-FTSTS } \\
\text {-FRT } \\
\text {-Maximal vertical jump test }\end{array}$ & $\begin{array}{l}\text { Protocol duration: } 8 \text { months } \\
\text { Frequency: } 2 \text { / week } \\
\text { Session: } 30 \text { min } \\
\text { IG: Supervised } \\
\text { First month: Body weight and low-load } \\
\text { exercise variants, with focus on progressively } \\
\text { learning the movement patterns. } \\
4 \text { fundamental exercise within } 2 \text { months } \\
\text { Remainder intervention period: } \\
\text { Resistance exercise: deadlift, overhead press, } \\
\text { back squat } \\
\text { Warm-up: } 2 \text { sets of deadlift at } 50 \% \text { to } 7 \% 1 \mathrm{RM} \\
\text { Reps: } 5 \text { sets of } 5 \text { repetition } \\
\text { Intensity: }>80 \% \text { to } 85 \% 1 \text { RM } \\
\text { CG: Home-based } 8 \text {-month, twice-weekly, } \\
30-\text { min } \\
\text { Warm-up: walking (10 min) } \\
\text { Cool down (5 min) } \\
\text { Resistance exercise: low-load resistance } \\
\text { training (lunges, calf raises, standing forward } \\
\text { raise, and shrugs) } \\
\text { Stretching: side-to-side neck stretch, static } \\
\text { calf stretch, shoulder stretch, and side-to-side } \\
\text { lumbar spine stretch. } \\
\text { Reps: } 10 \text { to } 15 \\
\text { Intensity: }<60 \% 1 \text { RM } \\
\text { Drug exposure (\% allocated subject): IG } 100 \% \text {; } \\
\text { CG 100\% (Bisphosphonates, calcium and } \\
\text { vitamin D) }\end{array}$ & 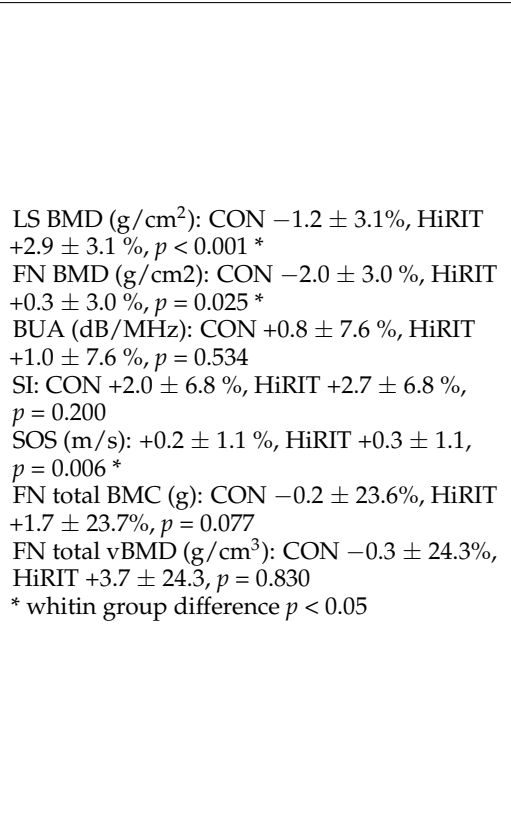 & $\begin{array}{l}\text { LIFTMOR trial showed a brief, } \\
\text { supervised, twice-weekly } \\
\text { HiRIT exercise intervention } \\
\text { was efficacious and superior to } \\
\text { previous programs for } \\
\text { enhancing bone at clinically } \\
\text { relevant sites, as well as stature } \\
\text { and functional performance of } \\
\text { relevance to falls in } \\
\text { postmenopausal women with } \\
\text { low to very low bone mass }\end{array}$ & $7 / 10$ \\
\hline $\begin{array}{l}\text { Borba-Pinheiro et al. } \\
\text { (2016) [14] }\end{array}$ & $\begin{array}{l}n=52 \text { women } \\
\text { Inclusion criteria } \\
\text { - Female volunteers } \\
\text {-Aged over } 50 \text { years } \\
\text {-Low BMD: T-score <1SD (low } \\
\text { bone density) } \\
\text {-Different ethnic population } \\
\text { (descendants of Europeans, } \\
\text { Blacks and Indians) } \\
\text {-Patient being treated with } \\
\text { sodium alendronate [70 mg] } \\
\text { and/or vitamin D3 } \\
\text {-No previous history of } \\
\text { fractures } \\
\text {-No history for at least } 1 \text { year of } \\
\text { regular practice of physical } \\
\text { activity } \\
\text {-Indication/medical clearance } \\
\text { for physical exercises practice. }\end{array}$ & $\begin{array}{l}\text { BMD } \\
\text { DXA } \\
\text { Functional autonomy } \\
\text { Latin American Development } \\
\text { Group for Maturity (GDLAM): } \\
\text { - 10-m walk (10MW) } \\
\text {-Rising from sitting position } \\
\text { (RSP) } \\
\text {-Rising frorm ventral decubitus } \\
\text { position (RVDP) } \\
\text {-Rising from a chair and } \\
\text { walking around the house } \\
\text { (RCWH) } \\
\text {-Putting on and take off a shirt } \\
\text { (PRTS) } \\
\text { Muscular Strength } \\
\text { 10 maximum repetitions test } \\
\text { (10MR) } \\
\text { Quality of life } \\
\text { Osetoporosis Assessment } \\
\text { Questionnaire (OPAQ) }\end{array}$ & $\begin{array}{l}\text { Protocol duration: } 13 \text { months } \\
\text { Session: } 60 \text { min } \\
\text { IG: RT3 ( } 3 \text { times a week) CG: RT2 (two times } \\
\text { a week) } \\
\text { Exercises: leg press } 45^{\circ} \text {; knee extension; } \\
\text { plantar flexion; squats; hip adduction; gluts } \\
\text { (machine for gluts); elbow flexion; elbow } \\
\text { extension; shoulder adduction } \\
\text { Posology: } 3 \text { sets per exercise. Repetitions } \\
\text { numers/sets, rest intervals between exercises } \\
\text { and sessions respected the scientific principle } \\
\text { of inter-dependence volume x intensity } \\
\text { (American College Sports Medicine). } \\
\text { Intensity: between } 60 \% \text { and } 90 \% \text {; months } \\
\text { cycles (60\%, } 65 \%, 70 \%, 75 \%, 80 \%, 85 \% \text { and } \\
90 \% \text { ); in addition } 3 \text { bimonthly cycles (70\%, } \\
80 \% \text {, } 90 \% \text { ) } \\
\text { Drug exposure (\% allocated subject): IG } 100 \% \text {; } \\
\text { CG 100\% (Sodium alendronate [70 mg] } \\
\text { and/or vitamin D3) }\end{array}$ & 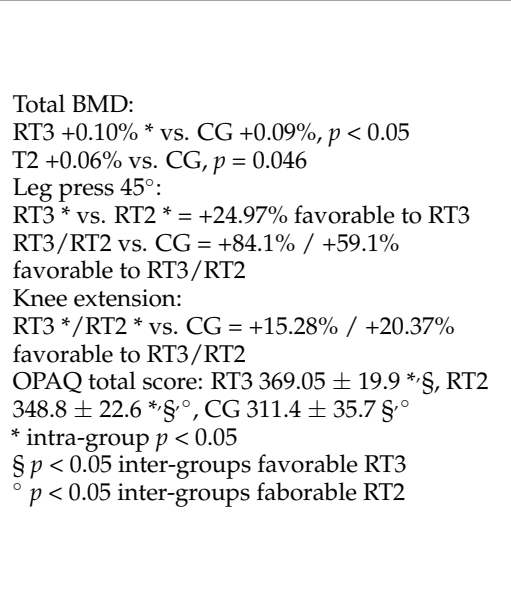 & $\begin{array}{l}\text { Both experimental groups } \\
\text { presented favorable results for } \\
\text { BMD, strength, FA and QoL. } \\
\text { However, the RT3 showed the } \\
\text { best results compared to other } \\
\text { groups atter } 13 \text { months of } \\
\text { intervention. }\end{array}$ & $8 / 10$ \\
\hline
\end{tabular}


Table 1. Cont.

\begin{tabular}{|c|c|c|c|c|c|c|}
\hline Author, Year & Participants & Outcome Measures & Intervention & Results & Conclusions & PEDro Score \\
\hline $\begin{array}{l}\text { Khalili et al. (2016) } \\
\text { [25] }\end{array}$ & $\begin{array}{l}n=183 \text { women } \\
\text { Inclusion criteria } \\
\text { - Women with primary OP } \\
\text { (with DEXA bone } \\
\text { densitometry) } \\
\text { - } 30 \text { to } 50 \text { degrees kyphosis } \\
\text { (with lateral standard wiew } \\
\text { thoracic spine x-ray graphs). }\end{array}$ & $\begin{array}{l}\text { Quality of life } \\
\text { - Persian version of the SF-36 } \\
\text { QOL questionnaire }\end{array}$ & $\begin{array}{l}\text { Protocol duration: } 6 \text { months } \\
\text { Session: } 30 \text { min } \\
\text { Frequency: } 5 \text { times a week } \\
\text { IG } \\
\text { Warm-up: Walk and back extensors } \\
\text { Resistance exercise: Home-base } \\
\text { Reps: } 10 \text { contractions of back extensor } \\
\text { without increasing the low back lordosis } \\
\text { Drug exposure (\% allocated subject): IG 100\%; } \\
\text { CG 100\% (Calcium [1000 mg], vitamin D [800 } \\
\text { IU] daily, sodium alendronate [70 mg] } \\
\text { weekly) }\end{array}$ & $\begin{array}{l}\text { Phisical Component Score: } \\
\text { Intervention group } 270.55 \pm 58.72 * \text {, Control } \\
\text { group } 233.30 \pm 67.47 *, p=0.00 \\
\text { Mental Component Score: } \\
\text { Intervention group } 255.78 \pm 61.19 * \text {, Control } \\
\text { group 239.58 } \pm 73.60, p=0.39 \\
\text { * intra-group } p<0.05\end{array}$ & $\begin{array}{l}\text { Home-based exercise with no } \\
\text { direct supervision improved } \\
\text { QOL in elderly women whith } \\
\text { OP at a 6-month follow-up. }\end{array}$ & $8 / 10$ \\
\hline $\begin{array}{l}\text { Murtezani et al. (2014) } \\
\text { [15] }\end{array}$ & $\begin{array}{l}n=62 \text { women } \\
\text { Inclusion criteria } \\
\text { Women recently diagnosed } \\
\text { (within the past } 6 \text { months) with } \\
\text { OP on account of a DEXA scan } \\
\text { T score below }-2.5 \\
\text { Aged } 50-70 \text { years } \\
\text { No history of vertebral } \\
\text { fractures or lower extremities } \\
\text { fractures } \\
\text { No endoprostheses or fixation } \\
\text { materials } \\
\text { Capable of signing written } \\
\text { informed consent }\end{array}$ & $\begin{array}{l}\text { Muscle Strength } \\
\text {-GS } \\
\text {-QS } \\
\text { Flexibility } \\
\text { - BRPT } \\
\text { Balance } \\
\text { - Berg Balance Scale (BBS) } \\
\text { Gait time } \\
\text { - 6MWT } \\
\text { Pain } \\
\text { - VAS }\end{array}$ & $\begin{array}{l}\text { Protocol duration: } 10 \text { months } \\
\text { Session: } 55 \text { min } \\
\text { Frequency: } 3 \text { times a week } \\
\text { IG (Land exercise) } \\
\text { Warm-up: } 10 \text { min. Stretching and balance } \\
\text { exercise at } 70-80 \% \\
\text { Exercises: leg press } 45^{\circ} \text {; knee extension; } \\
\text { plantar flexion; squats; hip adduction; gluts } \\
\text { (machine for gluts); elbow flexion; elbow } \\
\text { extension; shoulder adduction } \\
\text { Posology: } 3 \text { sets per exercise. Repetitions } \\
\text { numers/sets, rest intervals between exercises } \\
\text { and sessions respected the scientific principle } \\
\text { of inter-dependence volume x intensity } \\
\text { (American College Sports Medicine). } \\
\text { Intensity: between } 60 \% \text { and } 90 \% \text {; } 7 \text { months } \\
\text { cycles (60\%, } 65 \% \text {, } 70 \% \text {, } 75 \%, 80 \%, 85 \% \text { and } \\
90 \%) ; \text { in addition } 3 \text { bimonthly cycles ( } 70 \% \text {, } \\
80 \%, 90 \% \text { ) } \\
\text { Drug exposure (\% allocated subject): IG 100\%; } \\
\text { CG } 100 \% \text { (Dietary restriction and } \\
\text { supplementation (Calcium } 1000 \mathrm{mg} \text { ] daily } \\
\text { and Vitamin D [800-1000 IU] daily) }\end{array}$ & $\begin{array}{l}\text { VAS: LE }-81.26 \% * \text {, Control }-32.28 \%, \\
p<0.001 \\
\text { GS: LE }-4.54 \% * \text {, Control }-2.35 \%, p=0.002 \\
\text { QS: LE }+4.4 \% * \text {, Control }+1.1 \% *, p=0.002 \\
\text { BBS: LE }+3.24 \% * \text {, Control }+3.04 \%, p=0.38 \\
\text { 6MWT: LE }+18.72 \% * \text {, Control }+12.29 \% * \text {, } \\
p<0.001 \\
\text { BMD: LE }+5.35 \% * \text {, Control }+3.92 \%, p<0.001 \\
\text { T-score: LE }-12.04 \% * \text {, Control }-6.44 \%, \\
p<0.001 \\
{ }^{*} \text { whitin group difference } p<0.05\end{array}$ & $\begin{array}{l}\text { Significant improvements in } \\
\text { physical function and BMD } \\
\text { suggest that land exercise is a } \\
\text { possible alterative for } \\
\text { postmenopausal women with } \\
\text { OP. }\end{array}$ & $6 / 10$ \\
\hline Mosti et al. (2013) [11] & $\begin{array}{l}n=16 \text { women } \\
\text { Inclusion criteria } \\
\text { At least } 2 \text { years } \\
\text { postmenopausal } \\
\text { Age }<75 \text { years old } \\
\text { BMD t-score between }-1.5 \text { and } \\
-4.0 \text { at the lumbar spine, } \\
\text { femoral neck or total hip }\end{array}$ & $\begin{array}{l}\text { MS, RFD and PF } \\
\text { - Squat exercise machine } \\
\text { BMC and BMD } \\
\text { - Lumbar spine } \\
\text { - Femoral neck } \\
\text { - Total hip } \\
\text { Vitamin D and Markers of Bone } \\
\text { Metabolism } \\
\text { Treadmill Testing } \\
\text { - Peak oxygen consumption } \\
\text { (VO } 2 \text { peak) }\end{array}$ & $\begin{array}{l}\text { Protocol duration: } 12 \text { weeks } \\
\text { Frequency: } 3 \text { times a week } \\
\text { IG: (Maximal Strength Training MST) } \\
\text { Workout: supervised maximal strength } \\
\text { training, focused on high acceleration during } \\
\text { the concentric phase, resulting in a high RFD } \\
\text { during muscle contraction. } \\
\text { Posology: Each set was separated by } 2-3 \text { min } \\
\text { rest. } \\
\text { Intensity: If the participants could perform }>5 \\
\text { repetitions, the training load was increased } \\
\text { by } 2.5 \mathrm{~kg} \text {. } \\
\text { Drug exposure (\% allocated subject): IG } 100 \% \text {; } \\
\text { CG } 100 \% \text { (Calcium and Vitamin D) }\end{array}$ & $\begin{array}{l}\text { 1RM }(\mathrm{kg}) \text { : TG } 93.13 \pm 8.10 *, \text { CG } 62.19 \pm \\
14.36 \\
\text { Dynamic RFD (N/s): TG } 1103.35 \pm 282.75 * \\
\text { CG } 1386.02 \pm 595.00 \\
\text { Peak force (N): TG } 1397.23 \pm 123.84 * \\
\text { CG } 1389 \pm 260.00 \\
\text { BMC (g): TG lumbar }+2.9 \pm 2.8 \%(p=0.012) \text {; } \\
\text { femoral neck }+4.9 \% \pm 5.6 \%(p=0.043), \text { No } \\
\text { change in CG } \\
\text { Serum bone markers: } \\
\text { - Vitamin D (nmol/L) TG } 80.7 \pm 29.2 ; \text { CG } 99.5 \\
\pm 16.5) \text {. } \\
\text { P1NP and CTX no significant changes } \\
\text { P1NP/CTX ratio TG }+21.5 \pm 40.5 \%, p=0.093 \\
{ }^{*} \text { Difference within group, } p<0.05 \\
{ }^{\circ} \text { Difference between group } p<0.01\end{array}$ & $\begin{array}{l}\text { This study demonstrates that } \\
\text { squat exercise MST, applying } \\
\text { only one exercise, improves } \\
\text { 1RM, RFD, and BMC in } \\
\text { patients with OP and } \\
\text { osteopenia. }\end{array}$ & $6 / 10$ \\
\hline
\end{tabular}


Table 1. Cont.

\begin{tabular}{|c|c|c|c|c|c|c|}
\hline Author, Year & Participants & Outcome Measures & Intervention & Results & Conclusions & PEDro Score \\
\hline $\begin{array}{l}\text { Marchese et al. (2012) } \\
\text { [12] }\end{array}$ & $\begin{array}{l}n=22 \text { women } \\
\text { Inclusion criteria } \\
\text { Diagnosis of osteopenia by } \\
\text { DXA performed within } 6 \\
\text { months } \\
\text { Age between } 40 \text { and } 80 \text { years } \\
\text { old }\end{array}$ & $\begin{array}{l}\text { BMD } \\
\text { - Lumbar spine } \\
\text { - Proximal femoral epiphysis } \\
\text { Osteocalcin and CTX in serum } \\
\text { Electromyographic signal } \\
\text { - Quadriceps femoris } \\
\text { - Hip adductors } \\
\text { - Adbominal } \\
\text { - Paravertebral } \\
\text { Static Balance } \\
\text { - LC } \\
\text { - MAO } \\
\text { 6MWT } \\
\text { Disability and Quality of Life } \\
\text { - EuroQoL }\end{array}$ & $\begin{array}{l}\text { Protocol duration: } 24 \text { weeks } \\
\text { Frequency: } 3 \text { times a week } \\
\text { Session: } 00 \text { min } \\
\text { IG: Training group } \\
\text { A combination of exercised designed to } \\
\text { improve strength and muscle tropism, } \\
\text { aerobic capacity, coordination and balance, } \\
\text { designed to stimulate bone tissue in an } \\
\text { atypical and intermittent compression, } \\
\text { bending and tensile multi-directional stress. } \\
\text { Drug exposure (\% allocated subject): IG } \\
18.1 \% \text {; CG } 27.2 \% \text { (Antiresorptives) }\end{array}$ & 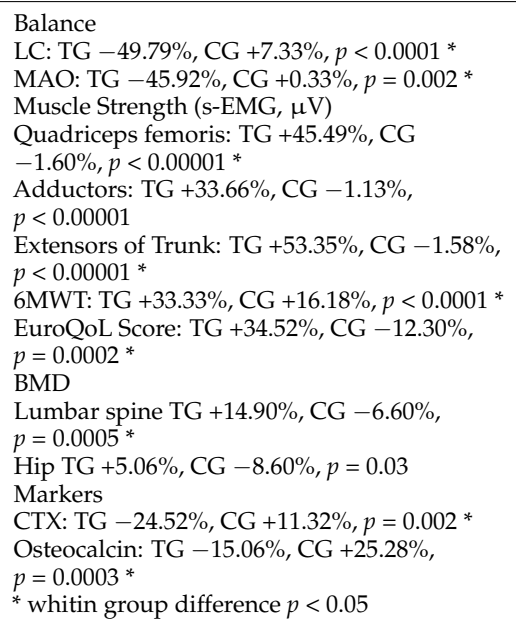 & $\begin{array}{l}\text { A improve strength and muscle } \\
\text { tropism, coordination and } \\
\text { balance, can provide } \\
\text { advantages of unquestioned } \\
\text { importance in bone mass, } \\
\text { neuromuscular function, } \\
\text { reduced risk of falling and } \\
\text { general health.rehabilitation } \\
\text { program of group exercise } \\
\text { based on gravitational load, } \\
\text { designed to }\end{array}$ & $5 / 10$ \\
\hline Burke et al. (2012) [26] & $\begin{array}{l}n=33 \text { women } \\
\text { Inclusion criteria } \\
\text { Women from } 65 \text { to } 79 \text { year of } \\
\text { age } \\
\text { Diagnosis of OP (according to } \\
\text { the WHO criteria) } \\
\text { BMD reduced at leat } 2.5 \mathrm{SD} \\
\text { compared with young adults } \\
\text { (region of lumbar spine) }\end{array}$ & $\begin{array}{l}\text { Postural control } \\
\text { LOS } \\
\text { CTSIBm } \\
\text { Inferior Members Strength } \\
\text { (Isometric Strength) } \\
\text { Ankle dorsiflexion } \\
\text { Knee extension } \\
\text { Kn } \\
\text { ee flexion }\end{array}$ & $\begin{array}{l}\text { Protocol duration: } 8 \text { weeks } \\
\text { Frequency: } 2 \text { times a week } \\
\text { Session: } 60 \text { min } \\
\text { IG (Strength group) } \\
\text { Warm-up: } 10 \text { min walking at low intensity } \\
\text { Exercises: } \\
\text { Balance exercise ( } 20 \text { min): walking in the } \\
\text { tandem position, on the tips the toes and heel, } \\
\text { sideways, while raising the leg and } \\
\text { controlateral arm; standing on one leg, in the } \\
\text { tandem position; } \\
\text { Strengthening exercises for lower limb ( } 30 \\
\text { min): exercise for knee extensor muscle, hip } \\
\text { flexors muscles and akle extensor muscles. } \\
\text { Posology: } 10 \text { repetitions, } 1 \text { min between sets. } \\
\text { CG1 (Stretching group) } \\
\text { CG2 (Education) } \\
\text { Drug exposure (\% allocated subject): IG } 94 \% \text {; } \\
\text { CG1 52\%; CG2 } 56 \% \text { (Medication and calcium } \\
\text { supplementation) }\end{array}$ & $\begin{array}{l}\text { Adherence: } 82.3 \% \\
\text { Isometric strength: } \\
\text { Ankle flexion IG }+4.4 \mathrm{~kg}, \mathrm{CG}+0.3 \mathrm{~kg}, \\
p=0.012^{*} \\
\text { Knee extension IG }+4.43 \mathrm{~kg}, \mathrm{CG}+0.1 \mathrm{~kg}, \\
p=0.003^{*} \\
\text { Knee flexion IG }+1.71 \mathrm{~kg}, \mathrm{CG}+0.22 \mathrm{~kg}, \\
p=0.003^{*} \\
\text { Postural control: } \\
\text { COP velocity IG }+2.34^{\circ} / \mathrm{s}, \mathrm{CG} 0.01^{\circ} / \mathrm{s}, \\
p=0.009^{*} \\
\text { Directional control IG }+5.34 \%, \mathrm{CG} 0.44 \% \text {, } \\
p=0.002^{*} \\
\mathrm{CTSIBm}^{*} \text { (closed eyes) IG }-0.21^{\circ} / \mathrm{s}, \mathrm{CG} \\
+0.05^{\circ} / \mathrm{s}, p=0.021^{*} \\
{ }^{*} \text { whitin group difference } p<0.05\end{array}$ & $\begin{array}{l}\text { Our study suggests that, in old } \\
\text { woman with OP, } 8 \text { weeks of } \\
\text { exercises improving balance } \\
\text { and inferior member strength } \\
\text { yielded improvement of } \\
\text { postural control and of } \\
\text { muscular strength. }\end{array}$ & $6 / 10$ \\
\hline
\end{tabular}


Table 1. Cont.

\begin{tabular}{|c|c|c|c|c|c|c|}
\hline Author, Year & Participants & Outcome Measures & Intervention & Results & Conclusions & PEDro Score \\
\hline $\begin{array}{l}\text { Borba-Pinheiro et al. } \\
\text { (2010) [18] }\end{array}$ & $\begin{array}{l}n=28 \text { women } \\
\text { Inclusion criteria } \\
\text { Women with OP and/or } \\
\text { osteopenia in at least one of the } \\
\text { measurements of BMD T-score } \\
\text { Patients treated with sodium } \\
\text { alendronate ( } 70 \mathrm{mg}) \\
\text { No history of fractures } \\
\text { No history for at least } 1 \text { year of } \\
\text { regular practice of physical } \\
\text { activity } \\
\text { Good physical and mental } \\
\text { health }\end{array}$ & $\begin{array}{l}\text { BMD } \\
\text { - Lumbar spine } \\
\text { - Proximal femur } \\
\text { Body balance } \\
\text { - Static Balance Test with Visual } \\
\text { Control } \\
\text { Quality of Life } \\
\text { - OPAQ }\end{array}$ & $\begin{array}{l}\text { Protocol duration: } 12 \text { months } \\
\text { Frequency: } 3 \text { times a week } \\
\text { Session: } 60 \text { min } \\
\text { IG: RTG } \\
\text { Exercises: leg press } 45^{\circ} \text {; knee extension; } \\
\text { plantar flexion; squats; hip adduction; gluts } \\
\text { (machine for gluts); elbow flexion; elbow } \\
\text { extension; shoulder adduction } \\
\text { Posology: } 10 \text { maximum repetitions (10RM) } \\
\text { test } \\
\text { Intensity: } 70-90 \% \\
\text { CG1: JUG } \\
\text { Exercises: Traditional methodology for judo } \\
\text { classes } \\
\text { CG2: WAG } \\
\text { Exercises: in a } 25-m \text { pool, } 1.45 \mathrm{~m} \text { deep; } \\
\text { dislocations (previous, posterior and lateral), } \\
\text { shoulder adduction/abduction, short jumps } \\
\text { with knee extension, alternate elbow flexion, } \\
\text { alternate knee flexion, alternate elbow } \\
\text { extension, hip adduction/abduction, } \\
\text { shoulder abduction/adduction, squats. } \\
\text { Drug exposure (\% allocated subject): IG 100\%; } \\
\text { CG 100\% (Sodium alendronate [70 mg] } \\
\text { weekly) }\end{array}$ & $\begin{array}{l}\text { BMD } \\
\text { Lumbar: RTG } 0.091, \text { JUG } 0.079 \text {, WUG } 0.034 \text {, } \\
\text { CG }-0.024, p=0.002, p=0.003, \mathrm{~ns} \\
\text { Neck of femur: RTG } 0.083, \text { JUG } 0.019 \text {, WUG } \\
-0.007, \text { CG }-0.06, p=0.002, \text { ns, ns } \\
\text { Great trochanter: RTG } 0.049, \text { JUG } 0.015 \text {, } \\
\text { WUG, } 0.018 \text {, CG }-0.029, p=0.002 \text {, ns, ns } \\
\text { Body balance } \\
\text { RTG 5.74, JUG 5.30, WUG 0.018, CG } \\
-1.06, p=0.018, p=0.022 \text {, ns } \\
\text { OPAQ } \\
\text { RTG 30.56, JUG 53.09, WUG 7.63, CG }-7.29 \text {, } \\
p=0.006, p=0.000, \text { ns } \\
{ }^{*} \text { whitin group difference } p<0.05\end{array}$ & $\begin{array}{l}\text { The type of physical activity } \\
\text { examined in this study could } \\
\text { be raccomended alone or as } \\
\text { adjunvtive therapy to a } \\
\text { biphosponate in } \\
\text { postmenopausal women with } \\
\text { low BMD, especially resistance } \\
\text { training. }\end{array}$ & $5 / 10$ \\
\hline
\end{tabular}


Table 1. Cont.

\begin{tabular}{|c|c|c|c|c|c|c|}
\hline Author, Year & Participants & Outcome Measures & Intervention & Results & Conclusions & PEDro Score \\
\hline $\begin{array}{l}\text { Bocalini et al. (2009) } \\
\text { [17] }\end{array}$ & $\begin{array}{l}n=35 \text { women } \\
\text { Inclusion criteria } \\
\text { Women older than } 55 \text { years } \\
\text { Able to train } 3 \text { times per week } \\
\text { in the course of } 24 \text { weeks of the } \\
\text { protocol }\end{array}$ & $\begin{array}{l}\text { Body composition } \\
\text { BMI } \\
\text { Body fat percentage } \\
\text { BMD } \\
\text { - Lumbar spine } \\
\text { - Femur neck } \\
\text { Muscle Strength (1RM) } \\
\text { - Chest press } \\
\text { - Leg extension }\end{array}$ & $\begin{array}{l}\text { Protocol duration: } 24 \text { weeks } \\
\text { Frequency: } 3 \text { times a week } \\
\text { Session: } 1 \text { h supervised } \\
\text { IG } \\
\text { Warm-up: } 10 \text { min of running with low impact } \\
\text { at } 50 \% \text { of maximum hearth rate; } 1 \text { set at } 50 \% \\
\text { 1RM } \\
\text { Workout (TR): Focus on eccentric muscle } \\
\text { action. Leg press, chest press, leg curl, } \\
\text { latissumus pull down, elbow flexion, elbow } \\
\text { extension, leg extension, upper back row, } \\
\text { military press, hip abductor, hip adductor, } \\
\text { abdominal curls. } \\
\text { Drug exposure (\% allocated subject): IG } 100 \% \text {; } \\
\text { CG } 100 \% \text { (Antiresorptives) }\end{array}$ & $\begin{array}{l}\text { MS: } \\
\text { TR } 62 \pm 5 \mathrm{~kg},+39 \%, p<0.001 \text { lower limb; } 37 \\
\pm 6 \mathrm{~kg},+46 \%, p<0.001 \text { upper body } \\
\text { UN } 38 \pm 7 \mathrm{~kg},-2.5 \%, p>0.05 \text { lower limb; } \\
23.5 \pm 5 \mathrm{~kg},+4.5 \%, p>0.05 \text { upper body } \\
\text { BMD: } \\
\text { TR } 0.880 \pm 0.001 \mathrm{~g} / \mathrm{cm}^{2}, p>0.05 \text { lumbar } \\
\text { spine, } 0.704 \pm 0.001 \mathrm{~g} / \mathrm{cm}^{2} \text { femoral neck } \\
\text { UN } 0.873 \pm 0.002 \mathrm{~g} / \mathrm{cm}^{2}, p<0.05 \text { lumbar } \\
\text { spine, } 0.695 \pm 0.001 \mathrm{~g} / \mathrm{cm}^{2} \text { femoral neck }\end{array}$ & $\begin{array}{l}\text { We demonstrated the positive } \\
\text { effects of strength training on } \\
\text { the body composition } \\
\text { parameters, muscular strength, } \\
\text { and bone health of } \\
\text { postmenopausal women } \\
\text { without hormone replacement } \\
\text { therapy. }\end{array}$ & $6 / 10$ \\
\hline $\begin{array}{l}\text { Tolomio et al. (2008) } \\
\text { [16] }\end{array}$ & $\begin{array}{l}n=64 \text { women } \\
\text { Inclusion criteria } \\
\text { Postmenopausal women (age } \\
\text { between } 50 \text { and } 70 \text { years) } \\
\text { Diagnosis of osteopenia or OP } \\
\text { ( } t \text {-score determined by } \\
\text { ultrasounds }<1.0 S D \\
\text { Lack of any disease that affect } \\
\text { bone metabolism } \\
\text { No previous skeletal fractures } \\
\text { Lack of any controindication to } \\
\text { perform physical activity }\end{array}$ & $\begin{array}{l}\text { Bone quality } \\
\text { Phalangeal quantitative } \\
\text { osteosonography } \\
\text { As-so } \\
\text { UBPS } \\
\text { Muscle Strength (1RM) } \\
\text { - Knee extensor muscles }\end{array}$ & $\begin{array}{l}\text { Protocol duration: } 20 \text { weeks } \\
\text { Frequency: } 3 \text { times a week } \\
\text { Session: two } 60 \text {-min sessions and one } 45 \text {-min } \\
\text { session } \\
\text { IG } \\
60-m i n \text { session: } \\
\text { Warm-up: } 20-25 \text { min of walking, stretching, } \\
\text { small jumps. } \\
\text { Workout: } 30 \text {-min training; } \\
\text { callistheni/isometric exercises and exercises } \\
\text { with dumbells, Thera-Bands, balls aimed to } \\
\text { improve range of motion, overall Strength, } \\
\text { balance and aerobic capacity. } \\
\text { Cool down: }-10 \text { min; stretching, breathing, } \\
\text { postural exercises } \\
\text { Volume: graded increase of intensity and } \\
\text { number of rep/series starting after the fifth } \\
\text { week of training. } \\
\text { CG } \\
45-m i n \text { session: } \\
\text { Combination of aerobic endurance and } \\
\text { Strength exercises. } \\
\text { Workout: Circuit training of } 6 \text { bouts of } \\
\text { exercise lasting } 5 \text { min each; treadmill, leg } \\
\text { extension, arm ergometer, horizontal leg } \\
\text { press, bike, lat machine. Indication to } \\
\text { progressively increase repetitions or load } \\
\text { lifted in during each 5-min Strength exercise. } \\
\text { Drug exposure (\% allocated subject): IG } \\
58,6 \% \text {; CG: } 55 \% \text { (Bisphosphonates, calcium } \\
\text { and Raloxifene) }\end{array}$ & $\begin{array}{l}\text { Ad-Sos: EG } 1988.8 \pm 74.4 \mathrm{~m} / \mathrm{s}, p<0.05 ; \\
\text { CG ns } \\
\text { UBPS: EG } 36.8 \pm 21.3, p<0.05 ; \text { CG } 36.5 \pm \\
\text { 17.2, ns } \\
\text { T-score: EG }-2.1 \pm 1.1, p<0.05 ; \text { CG ns } \\
\text { Knee extension: } 52.7 \pm 9.5 \mathrm{~kg}, p<0.05 ; \text { CG ns }\end{array}$ & $\begin{array}{l}\text { In a group of postmenopausal } \\
\text { women, a supervised, } \\
\text { multidimensional exercise } \\
\text { program improved bone } \\
\text { quality, evaluated at the finger, } \\
\text { in a relatively short period of } \\
\text { time. }\end{array}$ & $6 / 10$ \\
\hline
\end{tabular}


Table 1. Cont.

Author, Year Participants Outcome Measures Intervention Results Conclusions PEDro Score

\section{Body composition \\ Protocol duration: 24 weeks Frequency: 3 times a week}

BM

FFM

FM

$\mathrm{SF}$

$\mathrm{VO}_{2}$

Inclusion criteri

No neuromuscular injury or

Brentano et al. (2008) engaged in any tipe Practiced sports occasionally at a ricet sports occas

Session: $1 \mathrm{~h}$ supervised

G. STG 2-4 sets for each exercises.
Nynamic Strength (1RM) Arm curl exercises Knee extension exercise
NVmetric Strength

Electromyographic Signal Vastus lateralis Vastus medialis BMD Lumbar spine Femur $\quad \mathrm{VO}_{2} \max$ and TE: increased significantly in Workout: leg press, hip abduction, hip both training group after 24 week fly, arm curl, tireps punificantly in STG and CTG, greater than the CON group.

The exercises were performed separately, with a 2-min rest between sets. Posology: 20-6 repetitions and 45-80\% 1 RM

CG: CTC

The exercises were performed with no rest

between exercise

Posology: $23-10$ repetitions and $45-60 \% 1 \mathrm{RM}$

$2-3$ sets for each exercise.

Drug exposure (\% allocated subject): IG 50\%
Isometric strength: Increased significantly in

both training group after 24 weeks

D. no alteration in BMD lumbar, BMD

neck, $\mathrm{BMD}$ inter, $\mathrm{BMD}$ troc, $\mathrm{BMD}$ ward in al

groups after the 24-week period.

Correlations:

CLS and $\mathrm{VO}_{2}$ max: $r=0.73, p=0.000$

LLS and TE: $r=0.72, p=0.000$

IS and $\mathrm{VO}_{2}$ max: $r=0.59, p<0$.
IS and TE: $r=0.54, p<0.01$ ircuit weight training is an effective training in strategy to

improve neuromuscular and

corditioning with no hispausal women training.

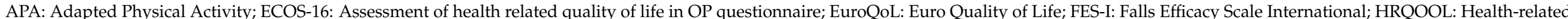

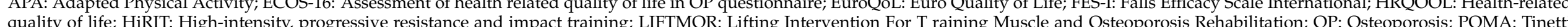

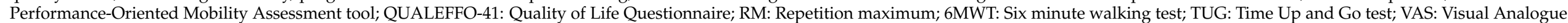

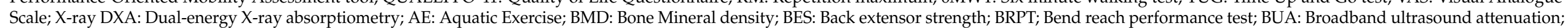

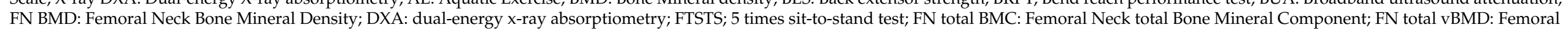

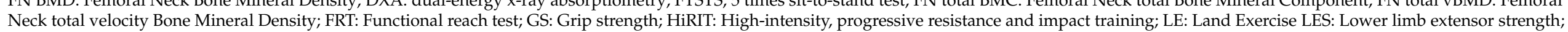

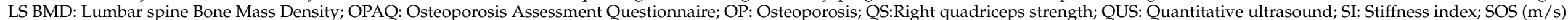

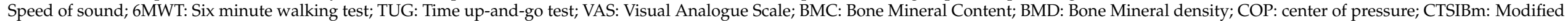

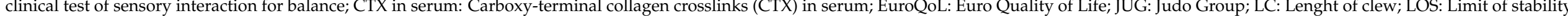

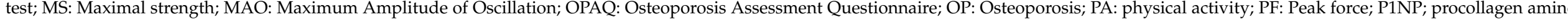

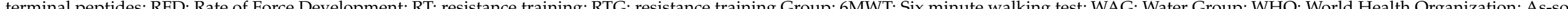

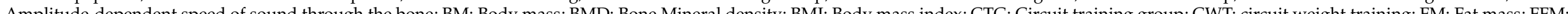

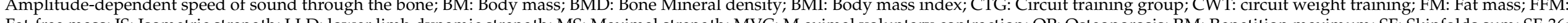
l high-intensity strength training; STG: Strength training group; TE: Time to Exhaustion in Exercise; TR: Trained Group; TUG: Time up-and-go test; UBPS: Ultrasound bone profile score; UN: Untrained GrouU+0070. 


\subsection{Association between Exercise Therapy and Muscular Strength}

In the included trials, muscle strength was evaluated at the level of back extensor strength, lower limb strength using leg press $45^{\circ}$, knee extension/flexion, ankle dorsiflexion, quadriceps strength (QS) and grip strength in the upper limb. The results agree in highlighting a significant correlation between an exercise protocol (Table 1) and muscle strength in all districts evaluated $[14,15,23,24,26]$.

\subsection{Association between Exercise Therapy and Balance}

The dynamic balance was evaluated, within the trials, using the Tinetti Scale and the Berg Balance Scale; the static one was evaluated using a stabilometric platform (Stabilometric platform E.P.S./R/LorAn Engineering, Bologna, Italy), analyzing the stakinesiogram and the stabiligram. For both components (dynamic and static) a significant correlation between exercise and balance improvements was found [12,14,15,18,21,27], (Table 1).

\subsection{Association between Exercise and Quality of Life}

Koevska et al [22] and Cergel et al [23] investigated this outcome using QUALEFFO-41; Borba-Pinheiro et al [18] used in both studies the OPAQ questionnaire, an instrument to measure the quality of life in patients with low BMD levels. In the other two trials in which this outcome was evaluated, the EQ-5D questionnaire was used, with the addition, in the study of Marini et al [21] of the ECOS-16, a specific tool for osteoporosis in assessing quality of life.

With the exception of Koevska et al [22], who reported only statistically significant changes within the sample study groups, the results of the other authors agree on a significant correlation between the execution of an exercise protocol (Table 1) and improvements in quality of life compared to those who do not perform any exercise program.

\subsection{Association between Exercise Therapy and Functionality}

The evaluation of physical performance was measured using different types of tests: time up-and-go test, 5 time sit-to-stand, 6-min walking test, functional reach test (FRT), bend reach performance test (BRPT), vertical jump (VJ) and chair sit-and-reach; then SF-36 in its physical function component and Tinetti's Scale were used.

The results report a statistically significant increase in performance in the evaluation tools used, with superiority of the groups performing an exercise protocol (Table 1) over the control groups [12,15,21,23-25,27].

\section{Discussion}

The purpose of this systematic review had to analyze the actual evidence about the muscle strengthening exercise and its efficacy in postmenopausal women suffering osteoporosis/osteopenia. The results of the studies analyzed, despite the wide range of years of publication, agree on the association between resistance exercise and its positive effect on the population examined in this review. However, the moderate-low level of methodological quality and the lack of homogeneity of the training programs analyzed suggest that there is contradictory evidence.

Strength training aims to promote osteogenesis in women diagnosed with OP, however, no significant changes are observed in vitamin-D levels, but significant changes are observed in bone architecture in both protein matrix and bone $(p=0.00177, p=0.00031)$ as well as in BMD. It seems that these changes would be more pronounced if the strength programs had a duration of 12 months [28,29]. However, the studies included in the present review presented a wide range of exercise duration (from 12 weeks for the program of Mosti et al. to 13 months for the program of Borba-Pinheiro et al.) with inconsistency in the positive impact on bone quality, which does not allow a firm conclusion on the optimal durability of the programs [14].

Muscle strengthening exercise improves other capacities such as isometric and isotonic strength of large neuromuscular complexes of both the lower and upper limbs that seem to 
be key in the primary prevention of falls [30]. Furthermore, these improvements are related to work intensity, showing that interventions are required that work at least at an intensity of 80 to $85 \% 1 \mathrm{RM}$ to achieve the desired effects. Effects on balance are also observed, although these improvements do not seem to extend beyond 10 months, so we believe it is necessary that this ability be introduced as early as possible in training programs, especially if the intensity is high at a lower frequency. However, this last aspect has not been rigorously demonstrated because for many researchers it would be difficult to justify the use of high intensity in frail women diagnosed with OP To overcome this procedural obstacle, Watson et al. proposed in their LIFTMOR study to divide the program into two mesocycles of 6 months duration, avoiding that excessive load accumulation ends up putting these patients at risk [24].

Regarding the topic of exercise parameters, a 2020 review and meta-analysis by Shojaa et al., aiming to analyse the effects of dynamic muscle strengthening exercise on BMD in postmenopausal women [31], showed no significant difference in BMD between protocols with different duration of intervention and between different exercise intensities. On the contrary, it showed a significant difference with a positive effect on bone quality, in favour of training with free weights and a low net training frequency ( $<2$ sessions/week).

On the other hand, it has been widely demonstrated that physical activity is able to promote bone formation, stimulating bone metabolism and its remodeling through mechanical loading (compression, tension and tissue shear) [32,33], improve hormonal regulation (estrogens, parathyroid hormone and glucocorticoids) [34-37] (with mimetic effect to hormone replacement therapy in postmenopausal women [38]), facilitate the regulation of signaling pathways [39-44], and stimulation of angiogenic-osteogenic responses [45]. However, and only from a clinical point of view, working in an aquatic environment may be a good approach to work in early phases due to the ease of working on the psychological and behavioral aspects associated with fear of movement.

Although not investigated in this review, considering the complexity and multifactorial nature of postmenopausal osteoporosis, further research is needed to investigate the possible synergistic effect of pharmacotherapy with certain exercise modalities, as highlighted in the study by Zhao et al. [46], who demonstrated that the combination of hormone replacement therapy (HRT) and a mixed-modality exercise protocol (high-impact activity in combination with high-intensity progressive muscle strengthening exercise was able to generate greater beneficial effects on hip and spine BMD in postmenopausal women than single-modality exercise.

With the results of this work, and given the absence of studies that address this question of clinical relevance, it is convenient to deepen the role of muscle strength training in the primary prevention of osteoporotic fracture in patients with osteopenia and even with delayed diagnosis of OP.

Furthermore, from a methodological point of view, differences have been detected in the proposed interventions, in the sample size and in the initial clinical status of the participants, which vary not only in issues such as age but also in the prognosis of their disease. Therefore, the existing variability and the lack of uniformity make it difficult to interpret and relate the different results, so it seems necessary to carry out more studies that assess suitability and promote the unification of criteria to achieve maximum effectiveness in therapeutic proposals to help us resolve the clinical question.

\section{Conclusions}

Muscle strengthening exercise in postmenopausal women with OP produces favorable results in terms of bone mineral density, strength, functionality, and quality of life. However, the benefits produced can be increased when combined with other therapeutic exercise modalities such as aerobic, balance and coordination.

Author Contributions: Conceptualization, S.M.P. and E.A.S.R.; methodology, J.H.V. and E.A.S.R.; software, J.H.V.; validation, all authors.; formal analysis, S.M.P., E.A.S.R. and J.L.A.P.; investigation, all authors.; resources, J.L.A.P.; data curation, S.M.P., E.A.S.R. and J.H.V.; writing-original draft 
preparation, E.A.S.R., S.M.P., J.L.A.P., A.B. and J.H.V.; writing-review and editing, E.A.S.R., S.M.P., A.B. and J.H.V.; visualization S.M.P. and J.L.A.P.; supervision, all authors.; project administration J.H.V. and E.A.S.R.; funding acquisition, J.L.A.P. All authors have read and agreed to the published version of the manuscript.

Funding: The publication of this work has been financed by the Universidad Europea de Canarias, C/Inocencio García 138300 La Orotava, 38300 Tenerife, Canary Islands, Spain.

Institutional Review Board Statement: Not applicable.

Informed Consent Statement: Not applicable.

Data Availability Statement: The data presented in this study are available on request from the corresponding authors.

Conflicts of Interest: The authors certify that they have no affiliations with or financial involvement in any organization or entity with a direct financial interest in the subject matter or materials discussed in the article.

\section{References}

1. Hernlund, E.; Svedbom, A.; Ivergard, M.; Compston, J.; Cooper, C.; Stenmark, J.; McCloskey, E.V.; Jonsson, B.; Kanis, J.A. Osteoporosis in the European Union: Medical management, epidemiology and economic burden. A report prepared in collaboration with the International Osteoporosis Foundation (IOF) and the European Federation of Pharmaceutical Industry Associations (EFPIA). Arch. Osteoporos. 2013, 8, 136. [CrossRef] [PubMed]

2. Kanis, J.A.; McCloskey, E.V.; Johansson, H.; Cooper, C.; Rizzoli, R.; Reginster, J.Y. European guidance for the diagnosis and management of osteoporosis in postmenopausal women. Osteoporos. Int. 2019, 30, 3-44. [CrossRef]

3. Riek, A.E.; Towler, D.A. The pharmacological management of osteoporosis. Mo. Med. 2011, 108, 118-123.

4. Benedetti, M.G.; Furlini, G.; Zati, A.; Mauro, G.L. The Effectiveness of Physical Exercise on Bone Density in Osteoporotic Patients. BioMed Res. Int. 2018, 2018, 4840531. [CrossRef]

5. Villafane, J.H.; Valdes, K.; Bertozzi, L.; Negrini, S. Minimal Clinically Important Difference of Grip and Pinch Strength in Women With Thumb Carpometacarpal Osteoarthritis When Compared to Healthy Subjects. Rehabil. Nurs. 2017, 42, 139-145. [CrossRef]

6. Chan, D.-C.; Chang, C.-B.; Han, D.-S.; Hong, C.-H.; Hwang, J.-S.; Tsai, K.-S.; Yang, R.-S. Effects of exercise improves muscle strength and fat mass in patients with high fracture risk: A randomized control trial. J. Formos. Med Assoc. Taiwan yi zhi. 2018, 117, 572-582. [CrossRef]

7. Pillastrini, P.; Ferrari, S.; Rattin, S.; Cupello, A.; Villafañe, J.H.; Vanti, C. Exercise and tropism of the multifidus muscle in low back pain: A short review. J. Phys. Ther. Sci. 2015, 27, 943-945. [CrossRef] [PubMed]

8. Ashe, M.C.; Gorman, E.; Khan, K.M.; Brasher, P.M.; Cooper, D.M.L.; McKay, H.A.; Liu-Ambrose, T. Does frequency of resistance training affect tibial cortical bone density in older women? A randomized controlled trial. Osteoporos. Int. 2013, $24,623-632$. [CrossRef] [PubMed]

9. Sanudo, B.; de Hoyo, M.; Del Pozo-Cruz, J.; Carrasco, L.; del Pozo-Cruz, B.; Tejero, S.; Firth, E. A systematic review of the exercise effect on bone health: The importance of assessing mechanical loading in perimenopausal and postmenopausal women. Menopause 2017, 24, 1208-1216. [CrossRef] [PubMed]

10. Asikainen, T.M.; Kukkonen-Harjula, K.; Miilunpalo, S. Exercise for health for early postmenopausal women: A systematic review of randomised controlled trials. Sports Med. 2004, 34, 753-778. [CrossRef]

11. Mosti, M.P.; Kaehler, N.; Stunes, A.K.; Hoff, J.; Syversen, U. Maximal strength training in postmenopausal women with osteoporosis or osteopenia. J. Strength Cond. Res. 2013, 27, 2879-2886. [CrossRef] [PubMed]

12. Marchese, D.; D'Andrea, M.; Ventura, V.; Montalcini, T.; Foti, D.; Pujia, A.; Gulletta, E.; Iocco, M. Effects of a Weight-Bearing Exercise Training on Bone Mineral Density and Neuromuscular Function of Osteopenic Women. Eur. J. Inflamm. 2012, 10, 427-435. [CrossRef]

13. Larpi, P.; Savanelli, A.; Cigliano, B.; De Fazio, P.; Vecchio, P.; Esposito, P. Aarskog's facio-digital-genital syndrome. Clinical contribution. Minerva Chir. 1985, 40, 1689-1692. [PubMed]

14. Borba-Pinheiro, C.J.; Dantas, E.H.M.; Vale, R.G.D.S.; Drigo, A.J.; Carvalho, M.C.G.D.A.; Tonini, T.; Meza, E.I.A.; de Figueiredo, N.M.A. Resistance training programs on bone related variables and functional independence of postmenopausal women in pharmacological treatment: A randomized controlled trial. Arch. Gerontol. Geriatr. 2016, 65, 36-44. [CrossRef] [PubMed]

15. Murtezani, A.; Nevzati, A.; Ibraimi, Z.; Sllamniku, S.; Meka, V.S.; Abazi, N. The effect of land versus aquatic exercise program on bone mineral density and physical function in postmenopausal women with osteoporosis: A randomized controlled trial. Ortop. Traumatol. Rehabil. 2014, 16, 319-325. [CrossRef]

16. Tolomio, S.; Ermolao, A.; Travain, G.; Zaccaria, M. Short-term adapted physical activity program improves bone quality in osteopenic/osteoporotic postmenopausal women. J. Phys. Act. Health 2008, 5, 844-853. [CrossRef]

17. Bocalini, D.S.; Serra, A.J.; dos Santos, L.; Murad, N.; Levy, R.F. Strength training preserves the bone mineral density of postmenopausal women without hormone replacement therapy. J Aging Health 2009, 21, 519-527. [CrossRef] [PubMed] 
18. Borba-Pinheiro, C.J.; de Alencar Carvalho, M.C.; da Silva, N.S.; Drigo, A.J.; Bezerra, J.C.; Dantas, E.H. Bone density, balance and quality of life of postmenopausal women taking alendronate participating in different physical activity programs. Ther. Adv. Musculoskelet Dis. 2010, 2, 175-185. [CrossRef]

19. Watson, S.L.; Weeks, B.K.; Weis, L.J.; Harding, A.T.; Horan, S.A.; Beck, B.R. High-intensity exercise did not cause vertebral fractures and improves thoracic kyphosis in postmenopausal women with low to very low bone mass: The LIFTMOR trial. Osteoporos Int. 2019, 30, 957-964. [CrossRef]

20. Brentano, M.A.; Cadore, E.L.; Da Silva, E.M.; Ambrosini, A.B.; Coertjens, M.; Petkowicz, R.; Viero, I.; Kruel, L.F.M. Physiological adaptations to strength and circuit training in postmenopausal women with bone loss. J. Strength Cond. Res. 2008, 22, 1816-1825. [CrossRef]

21. Marini, S.; Leoni, E.; Raggi, A.; Sanna, T.; Malavolta, N.; Angela, B.; Latessa, P.M.; Dallolio, L. Proposal of an Adapted Physical Activity Exercise Protocol for Women with Osteoporosis-Related Vertebral Fractures: A Pilot Study to Evaluate Feasibility, Safety, and Effectiveness. Int. J. Environ. Res. Public Health 2019, 16, 2562. [CrossRef]

22. Koevska, V.; Nikolikj-Dimitrova, E.; Mitrevska, B.; Gjeracaroska-Savevska, C.; Gocevska, M.; Kalcovska, B. Effect of Exercises on Quality of Life in Patients with Postmenopausal Osteoporosis-Randomized Trial. Open Access Maced. J. Med. Sci. 2019, 7, 1160-1165. [CrossRef]

23. Cergel, Y.; Topuz, O.; Alkan, H.; Sarsan, A.; Akkoyunlu, N.S. The effects of short-term back extensor strength training in postmenopausal osteoporotic women with vertebral fractures: Comparison of supervised and home exercise program. Arch. Osteoporos. 2019, 14, 82. [CrossRef]

24. Watson, S.L.; Weeks, B.K.; Weis, L.J.; Harding, A.; Horan, S.A.; Beck, B.R. High-Intensity Resistance and Impact Training Improves Bone Mineral Density and Physical Function in Postmenopausal Women With Osteopenia and Osteoporosis: The LIFTMOR Randomized Controlled Trial. J. Bone Miner. Res. 2018, 33, 211-220. [CrossRef] [PubMed]

25. Khalili, A.; Almasi, M.H.; Raeissadat, S.A.; Sedighipour, L.; Zamani, Y.S.; Zohoor, M.R.O. Long-term effects of back extensor strengthening exercises on quality of life in women with osteoporosis. J. Women Aging 2016, 29, 505-514. [CrossRef]

26. Burke, T.N.; Franca, F.J.; Ferreira de Meneses, S.R.; Ferreira de Meneses, S.R.; Cardoso, V.I.; Marques, A.P. Postural control in elderly persons with osteoporosis: Efficacy of an intervention program to improve balance and muscle strength: A randomized controlled trial. Am. J. Phys. Med. Rehabil. 2010, 89, 549-556. [CrossRef] [PubMed]

27. Teixeira, L.E.P.P.; Silva, K.N.G.; Imoto, A.M.; Teixeira, T.J.P.; Kayo, A.H.; Montenegro-Rodrigues, R.; Peccin, M.S.; Trevisani, V. Progressive load training for the quadriceps muscle associated with proprioception exercises for the prevention of falls in postmenopausal women with osteoporosis: A randomized controlled trial. Osteoporos. Int. 2010, 21, 589-596. [CrossRef]

28. Marin-Cascales, E.; Alcaraz, P.E.; Ramos-Campo, D.J.; Rubio-Arias, J.A. Effects of multicomponent training on lean and bone mass in postmenopausal and older women: A systematic review. Menopause 2018, 25, 346-356. [CrossRef]

29. Rhodes, E.C.; Martin, A.D.; E Taunton, J.; Donnelly, M.; Warren, J.; Elliot, J. Effects of one year of resistance training on the relation between muscular strength and bone density in elderly women. Br. J. Sports Med. 2000, 34, 18-22. [CrossRef] [PubMed]

30. Villafañe, J.H.; Bishop, M.D.; Pedersini, P.; Berjano, P. Physical Activity and Osteoarthritis: Update and Perspectives. Pain Med. 2019, 20, 1461-1463. [CrossRef]

31. Shojaa, M.; von Stengel, S.; Kohl, M.; Schoene, D.; Kemmler, W. Effects of dynamic resistance exercise on bone mineral density in postmenopausal women: A systematic review and meta-analysis with special emphasis on exercise parameters. Osteoporos. Int. 2020, 31, 1427-1444. [CrossRef] [PubMed]

32. Klein-Nulend, J.; Bacabac, R.G.; Bakker, A.D. Mechanical loading and how it affects bone cells: The role of the osteocyte cytoskeleton in maintaining our skeleton. Eur. Cells Mater. 2012, 24, 278-291. [CrossRef] [PubMed]

33. Zaidi, M. Skeletal remodeling in health and disease. Nat. Med. 2007, 13, 791-801. [CrossRef] [PubMed]

34. Krum, S.A. Direct transcriptional targets of sex steroid hormones in bone. J. Cell. Biochem. 2011, 112, 401-408. [CrossRef] [PubMed]

35. Guerrini, M.M.; Takayanagi, H. The immune system, bone and RANKL. Arch. Biochem. Biophys. 2014, 561, 118-123. [CrossRef]

36. Gardinier, J.D.; Mohamed, F.; Kohn, D.H. PTH Signaling During Exercise Contributes to Bone Adaptation. J. Bone Miner. Res. 2015, 30, 1053-1063. [CrossRef] [PubMed]

37. Gennari, L.; Merlotti, D.; Nuti, R. Selective estrogen receptor modulator (SERM) for the treatment of osteoporosis in postmenopausal women: Focus on lasofoxifene. Clin. Interv. Aging 2010, 5, 19-29. [CrossRef] [PubMed]

38. Bentz, A.T.; Schneider, C.M.; Westerlind, K.C. The relationship between physical activity and 2-hydroxyestrone, 16alphahydroxyestrone, and the 2/16 ratio in premenopausal women (United States). Cancer Causes Control 2005, 16, 455-461. [CrossRef]

39. Yuan, Y.; Zhang, L.; Tong, X.; Zhang, M.; Zhao, Y.; Guo, J.; Lei, L.; Chen, X.; Tickner, J.; Xu, J.; et al. Mechanical Stress Regulates Bone Metabolism Through MicroRNAs. J. Cell. Physiol. 2016, 232, 1239-1245. [CrossRef]

40. Mohamed, J.S.; Lopez, M.A.; Boriek, A.M. Mechanical stretch up-regulates microRNA-26a and induces human airway smooth muscle hypertrophy by suppressing glycogen synthase kinase-3beta. J. Biol. Chem. 2010, 285, 29336-29347. [CrossRef]

41. Shyu, K.-G.; Wang, B.-W.; Wu, G.-J.; Lin, C.-M.; Chang, H. Mechanical stretch via transforming growth factor-beta1 activates microRNA208a to regulate endoglin expression in cultured rat cardiac myoblasts. Eur. J. Heart Fail. 2013, 15, 36-45. [CrossRef] [PubMed]

42. Guo, Y.; Wang, Y.; Liu, Y.; Liu, Y.; Zeng, Q.; Zhao, Y.; Zhang, X.; Zhang, X. MicroRNA-218, microRNA-191*, microRNA-3070a and microRNA-33 are responsive to mechanical strain exerted on osteoblastic cells. Mol. Med. Rep. 2015, 12, 3033-3038. [CrossRef] 
43. Yehya, N.; Yerrapureddy, A.; Tobias, J.; Margulies, S.S. MicroRNA modulate alveolar epithelial response to cyclic stretch. BMC Genom. 2012, 13, 154. [CrossRef]

44. Yuan, Y.; Chen, X.; Zhang, L.; Wu, J.; Guo, J.; Zou, D.; Chen, B.; Sun, Z.; Shen, C.; Zou, J. The roles of exercise in bone remodeling and in prevention and treatment of osteoporosis. Prog. Biophys. Mol. Biol. 2016, 122, 122-130. [CrossRef] [PubMed]

45. Matsuzaki, H.; Wohl, G.R.; Novack, D.V.; Lynch, J.A.; Silva, M.J. Damaging fatigue loading stimulates increases in periosteal vascularity at sites of bone formation in the rat ulna. Calcif. Tissue Int. 2007, 80, 391-399. [CrossRef] [PubMed]

46. Zhao, R.; Xu, Z.; Zhao, M. Effects of Oestrogen Treatment on Skeletal Response to Exercise in the Hips and Spine in Postmenopausal Women: A Meta-Analysis. Sports Med. 2015, 45, 1163-1173. [CrossRef] [PubMed] 